\title{
Altered retrieval of melodic information in congenital amusia: insights from dynamic causal modeling of MEG data
}

\author{
Philippe Albouy ${ }^{1,2,3 *}$, Jérémie Mattout ${ }^{2}$, Gaëtan Sanchez ${ }^{2}$, Barbara Tillmann ${ }^{1 \dagger}$ and Anne Caclin ${ }^{2 \dagger}$ \\ 1 Lyon Neuroscience Research Center, Auditory Cognition and Psychoacoustics Team, CRNL, CNRS UMR5292, INSERM U1028; University Lyon 1, Lyon, France \\ ${ }^{2}$ Lyon Neuroscience Research Center, Brain Dynamics and Cognition Team, CRNL, CNRS UMR5292, INSERM U1028; University Lyon 1, Lyon, France \\ ${ }^{3}$ Montreal Neurological Institute, McGill University, Montreal, QC, Canada
}

\section{Edited by:}

Simone Dalla Bella, University of Montpellier 1, France

Reviewed by:

Nathan Weisz, University of Trento, Italy

Teppo Särkämö, University of Helsinki, Finland

*Correspondence:

Philippe Albouy, Montreal Neurological Institute, McGill University, 3801 University Street, Montreal, QC H3A 2B4, Canada e-mail:phv.albouy@gmail.com

${ }^{\dagger}$ These authors have contributed equally to this work.
Congenital amusia is a neuro-developmental disorder that primarily manifests as a difficulty in the perception and memory of pitch-based materials, including music. Recent findings have shown that the amusic brain exhibits altered functioning of a fronto-temporal network during pitch perception and short-term memory. Within this network, during the encoding of melodies, a decreased right backward frontal-to-temporal connectivity was reported in amusia, along with an abnormal connectivity within and between auditory cortices. The present study investigated whether connectivity patterns between these regions were affected during the short-term memory retrieval of melodies. Amusics and controls had to indicate whether sequences of six tones that were presented in pairs were the same or different. When melodies were different only one tone changed in the second melody. Brain responses to the changed tone in "Different" trials and to its equivalent (original) tone in "Same" trials were compared between groups using Dynamic Causal Modeling (DCM). DCM results confirmed that congenital amusia is characterized by an altered effective connectivity within and between the two auditory cortices during sound processing. Furthermore, right temporal-to-frontal message passing was altered in comparison to controls, with notably an increase in "Same" trials. An additional analysis in control participants emphasized that the detection of an unexpected event in the typically functioning brain is supported by right fronto-temporal connections. The results can be interpreted in a predictive coding framework as reflecting an abnormal prediction error sent by temporal auditory regions towards frontal areas in the amusic brain.

Keywords: tone deafness, effective connectivity, short-term memory, magneto-encephalography, pitch processing

\section{INTRODUCTION}

Congenital amusia refers to a neuro-developmental disorder characterized by impairments in pitch perception, production, and memory, more or less strongly accompanied by deficits along the time dimension (i.e., altered processing of rhythm or meter) (Ayotte et al., 2002; Peretz et al., 2002; Stewart, 2011; Peretz, 2013; Williamson and Stewart, 2013; Tillmann et al., 2015). The disorder cannot be explained by cognitive deficits, hearing loss, or brain damage (Ayotte et al., 2002; Peretz, 2013), and the pitchrelated impairments seem to impact mostly the processing of musical material, with some consequences also in the speech realm (Ayotte et al., 2002; Peretz and Hyde, 2003; Patel et al., 2005, 2008; Liu et al., 2010). Although the seminal studies on congenital amusia have focused on impairments of pitch discrimination and direction judgments (Ayotte et al., 2002; Peretz et al., 2002; Peretz and Hyde, 2003; Foxton et al., 2004; Hyde and Peretz, 2004; Stewart et al., 2006; Jones et al., 2009; Stewart, 2011; Jiang et al., 2013), subsequent studies have suggested that this disorder could also be traced down to deficits in short-term memory for pitch (Gosselin et al., 2009; Tillmann et al., 2009, 2015; Williamson and Stewart, 2010; Williamson et al., 2010; Albouy et al., 2013a,b).

The pitch-specificity of the short-term memory deficit has been observed with numerous behavioral approaches. Data collected with a conventional "span" memory task with numbers (Williamson and Stewart, 2010), or with a delayed comparison task with one-syllable words (Tillmann et al., 2009) showed intact performance in congenital amusics for verbal material, while confirming impaired performance for musical material. Additionally, in delayed comparison tasks, amusic individuals' performance is more strongly affected than control participants' performance when the durations of the retention interval between single tones are increased (Gosselin et al., 2009; Williamson et al., 2010) and the length of the to-be compared tone sequences are increased (Gosselin et al., 2009). Finally, amusic individuals exhibit increased sensitivity (i.e., leading to decreased performance) to interference caused by irrelevant tones presented during the 
retention interval (Gosselin et al., 2009; Williamson et al., 2010).

The functional cerebral correlates of the pitch memory deficit in congenital amusia have been investigated more recently. Recording Magnetoencephalography (MEG) signals during a delayed comparison task with melodies, functional abnormalities were revealed during encoding, short-term retention, and shortterm memory retrieval of the melodic information in amusic individuals as compared to the matched control participants who were typical non-musician listeners (Albouy et al., 2013a). During the encoding of melodies, the amusic brain elicited abnormal (decreased and delayed) N100m components in bilateral Inferior Frontal Gyri (IFG, pars opercularis) and auditory cortices. Abnormal functioning of fronto-temporal regions was also observed during other processing steps of the short-term memory task, as revealed by right-lateralized functional anomalies (in the Dorsolateral Prefrontal Cortex (DLPFC) and Posterior Parietal Cortex (PPC)) during the retention of pitch information, and by altered functioning of bilateral fronto-temporal (IFG, auditory cortex) regions during the short-term memory retrieval of melodies.

The functional abnormalities observed during a delayed comparison task with MEG recordings are in agreement with functional abnormalities observed during passive listening with fMRI (Hyde et al., 2011) and with anatomical abnormalities observed along the auditory-frontal pathway in the amusic brain (Hyde et al., 2006, 2007; Mandell et al., 2007; Loui et al., 2009). Overall, these findings were in agreement with data on the normal functioning (non-amusic) brain suggesting that pitch processing (perception and memory) involves both the auditory cortex and distant brain areas, notably frontal and parietal cortices (Griffiths, 1999; Griffiths et al., 1999; Maess et al., 2001; Janata et al., 2002a,b; Tillmann et al., 2003, 2006; Peretz and Zatorre, 2005; Koelsch et al., 2009; Foster and Zatorre, 2010; Schulze et al., 2011a,b; Schulze and Koelsch, 2012; Foster et al., 2013).

However, most of these studies described co-activation patterns of distant brain areas without analyzing the connectivity patterns within specific networks that are underlying participants' task performance. While different cognitive processes may recruit similar brain regions, the connectivity patterns between these regions can differ depending on the context of the operations being actively performed (D'Esposito, 2007). Indeed, beyond functional specialization, the principle of functional integration in cognitive neuroscience suggests that complex cognitive processes are supported by dynamic interactions between different brain areas (Varela et al., 2001; Friston et al., 2003; Garrido et al., 2007; Rauschecker and Scott, 2009). Understanding these dynamical interactions is relevant not only in the normally functioning brain, but also in impairments and deficits, such as amusia. While the implication of anomalies in the fronto-temporal regions in amusics' pitch deficits has now been reported in several studies, there remain open questions about whether and how altered connectivity patterns between these brain areas underlie the disorder.

Using functional connectivity measures on fMRI data during passive listening to pitch, Hyde et al. (2011) reported an increased lateral connectivity between the two auditory cortices and most importantly, a decreased connectivity between the right IFG and the right Superior Temporal Gyrus (STG) in the amusic brain in comparison to controls. This abnormal pattern of fronto-temporal connectivity has also been reported recently during resting state (Lévêque et al., submitted). Together with the anatomical abnormalities observed in this pathway with Diffusion Tensor Imaging (Loui et al., 2009), these findings led to the hypothesis that the cerebral correlates of congenital amusia could be related to decreased anatomical and functional fronto-temporal connectivity. However, although functional connectivity measures allow the establishment of statistical dependency between different brain regions, they do not provide information about the causal architecture of the interactions (Friston et al., 2003).

To improve the understanding of the effective connectivity patterns supporting auditory encoding of pitch information in the amusic brain, Albouy et al. (2013a) have used Dynamic Causal Modeling (DCM; David et al., 2006) of MEG data in a delayed comparison task. In this study, amusics' altered encoding of auditory information (i.e., during the first melody of the delayed comparison task) was related to (1) reduced intrinsic connectivity within each of the auditory cortices; (2) increased lateral connectivity between right and left auditory cortices; and (3) a decreased right frontal-to-temporal (backward) connectivity in amusics relative to controls. These results suggest that abnormal causal interactions underlie the altered brain responses observed within the auditory fronto-temporal network in the amusic brain. Moreover, these results suggest that in the typical brain, the encoding of auditory information, which allows keeping a memory trace of a previously presented stimulus in order to compare it (top-down) to a sound occurring later (bottom-up), implies a crucial role of backward connections (from the right IFG to the right auditory cortex) to support the construction of an appropriate memory trace of the stimulus.

Along these lines, the present study aims at (1) improving the characterization of the cerebral correlates of short-term memory deficits in congenital amusia; and (2) extending our understanding of the mechanisms supporting auditory shortterm memory in the typically functioning brain.

We here further analyzed the MEG data acquired in the study of Albouy et al. (2013a) where amusic participants and matched control participants performed a melodic contour task, in which two six-tone sequences had to be compared (same/different paradigm, also referred to as delayed comparison task). When the melodies were different, only one tone changed in the second melody. Focusing on this changed tone, Albouy et al. (2013a) have previously analyzed amplitude and source localization of Event Related Fields (ERFs) for the difference between "Different" and "Same" trials (for correct responses). While the control group showed activity in bilateral auditory cortices and in the pars opercularis of the IFG (BA 44) when they detect the changed tone, the amusic brain showed strongly reduced brain responses in these regions (see Albouy et al., 2013a).

Using data from the same experiment, we performed three types of DCM analyses investigating the ERFs during the second 
Table 1 | Demographic characteristics of participants and their data for behavioral pretests.

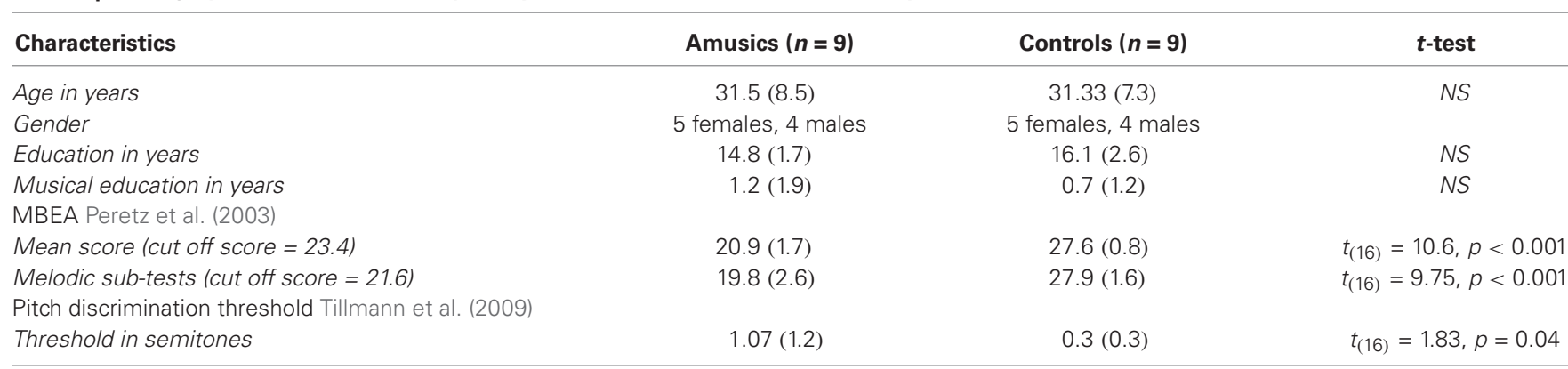

Educational background is calculated in years of education starting from the first year of primary school in the French system, at about 6 years of age. Results of the Montreal Battery of Evaluation of Amusia (MBEA) are expressed as number of correct responses (average over the six sub-tests of the battery, maximum score $=30$; and average over the three melodic subtests, maximum score $=30)$. Pitch Discrimination Threshold (PDT) scores are reported in semitones. Data are reported as a function of group, along with significance levels on corresponding t-tests; "NS" refers to a non-significant difference (p > 0.05). Standard deviations are in parentheses.

melody of the delayed comparison task: (1) By comparing amusics and controls for "Same" trials, we explored whether the previously reported anomalies in the amusic brain during the encoding of pitch (i.e., when processing the first melody of the pair in the delayed comparison task) are also present during shortterm memory retrieval (i.e., when processing the second melody of the pair); (2) By comparing "Different" and "Same" trials for controls only, we investigated what kind of connectivity patterns support the detection of a deviant tone in the typically functioning brain; and (3) By comparing amusics and controls for the "Difference Wave" ("Different" minus "Same" trials), we investigated whether amusics' altered brain responses related to the detection of the changed tone (short-term memory retrieval) could be also associated with abnormal connectivity patterns within this bilateral fronto-temporal network.

\section{METHODS}

In the present article, we performed new DCM analyses of the data from Albouy et al. (2013a). All details about the experiment (participants, materials, procedures, MEG pre-processing, and source reconstruction analyses) are described in Material and Methods of the original paper (from p. 1640-1646). We here present a short summary of the methods, and focus on the DCMs used to investigate the modulations of effective connectivity that support the short-term retrieval of pitch information.

\section{PARTICIPANTS}

Nine amusic individuals and nine matched non-musician controls participated to the study. In a previous testing session, all participants were tested with the Montreal Battery of Evaluation of Amusia (Peretz et al., 2003) and with a two-alternative forced-choice task (using a staircase procedure) to evaluate their pitch discrimination thresholds (Tillmann et al., 2009). Participants' demographic characteristics and data from pre-tests are presented in Table 1. Ethical approval was obtained from the French ethics committee on Human Research (CPP Sud-Est II, \#2006-018/A-1).

\section{DELAYED COMPARISON TASK AND PROCEDURE}

Participants performed a delayed comparison task for which they had to compare two six-tone sequences (S1, S2) separated by a silent retention period of $2000 \mathrm{~ms}$. The two tone sequences could be either the same or different. All sequences were composed of six $250-\mathrm{ms}$ piano tones presented successively without inter-stimulus-interval. One hundred and ninety two different melodies (sequences) were created using eight piano tones differing in pitch height (Cubase software, Steinberg); all used tones belonged to the key of C Major (C3, D3, E3, F3, G3, A3, B3, C4). These 192 sequences were used as S1. For S2 in "Different" trials, one tone (in positions 2-5) was replaced by a different tone of the set to create a contour-violation in the melody (Figure 1A). Participants performed the melodic task and another control task (not presented here, see Albouy et al., 2013a) during the MEG recording. Presentation software (Neurobehavioral systems, Albany, CA, USA) was used to run the experiment and to record button presses. For each trial, participants had to decide whether S2 was identical to S1 or different from S1. There were six blocks separated by $2-3 \mathrm{~min}$ of break. Note that there were also six blocks of the control task that were presented in alternation (counterbalanced across participants). Participants were informed of task order and asked to indicate their answers by pressing one of two keys with their right hand after the end of S2. They had $2 \mathrm{~s}$ to respond before the next trial occurring $2.5 \mathrm{~s}-3 \mathrm{~s}$ after the end of S2. No feedback was given during the experiment. In each block, 32 trials were presented (16 same pairs, 16 different pairs), resulting in 192 trials in total.

\section{MEG RECORDINGS AND ANALYSES}

The recordings were carried out using a 275-channel wholehead MEG system (CTF-275 by VSM Medtech Inc., Vancouver, Canada) with continuous sampling at a rate of $600 \mathrm{~Hz}$, a $0-150 \mathrm{~Hz}$ filter bandwidth, and first-order spatial gradient noise cancellation. Horizontal and vertical electrooculograms (EOG) and electrocardiogram were acquired with bipolar montages. Head position was determined with coils fixated at the nasion and the preauricular points (fiducial points, continuous 


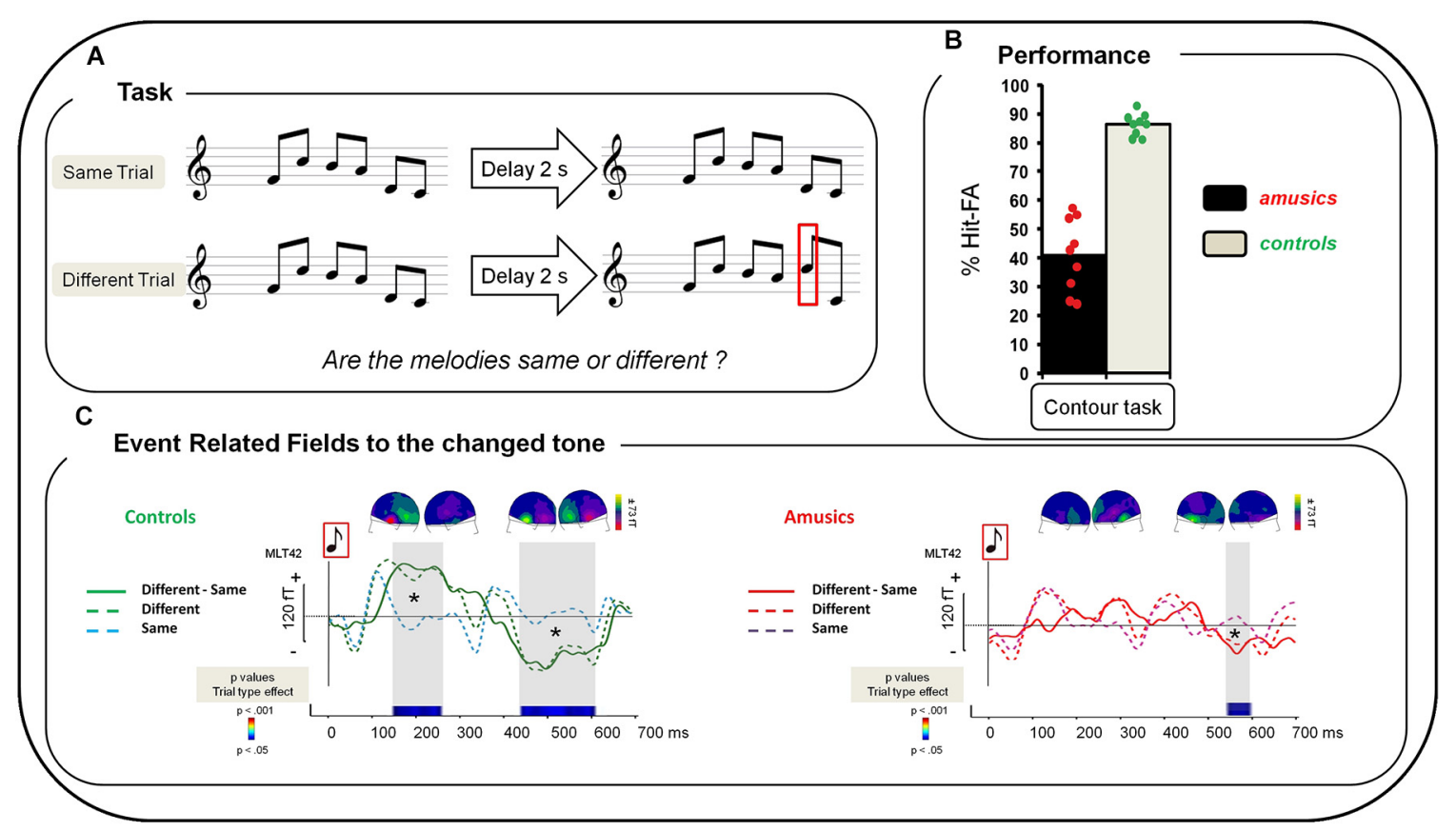

FIGURE 1 | (A) Examples of the musical stimuli. "Same" trials: After a $2 \mathrm{~s}$ delay, S1 was repeated as the second melody of the pair (S2). "Different" trials: one tone was changed in the second melody of the pair (red square). (B) Performance of amusic and control groups (Gray, Controls; Black, Amusics) in terms of percent of Hit-FA. Green circles: controls' individual performance; red circles: amusics' individual performance. (C) Grand average of a left temporal MEG sensor (MLT42) for a 0-700 ms time window after the onset of the changed tone in S2 for the Contour Task for each group and each type of trial. Left: For controls. Green dotted line: "Different" trials, correct responses; blue dotted line: "Same" trials, correct responses; green plain line: "Difference Wave" ("Different" trials— "Same" trials for correct responses). Right: For amusics. Red dotted line: "Different" Trials, correct responses; purple dotted line: "Same" trials, correct responses; red plain line: "Difference Wave" ("Different" trials—"Same" trials for correct responses). Sensor plots correspond to the mean event-related fields (ERFs) of the "Difference Wave" in the 150-250 ms and the 400-600 ms time-windows for the change tone (average of all participants of each group). Two-sample $t$-tests were performed at each time sample on sensor amplitudes in the 0-700 time window in the two groups of participants. $p$-values are reported across time in the lower panel with blue for $p<0.05$; green for $p<0.01$; and red for $p<0.001$. Note that only effects lasting longer than $15 \mathrm{~ms}$ were reported See Albouy et al. (2013a) for details. sampling at a rate of $150 \mathrm{~Hz}$ ). Participants were seated upright in a sound-attenuated, magnetically-shielded recording room, and listened to the sounds presented binaurally through airconducting tubes with foam ear tips. Prior to the MEG recording, participants' sound detection thresholds (using G3, the tone in the center of the tone set for S1) were determined for each ear, and the level was adjusted so that the sounds were presented at about 50-55 dB Sensation Level with a central position (stereo) with respect to the participant's head. MEG data were first analyzed in sensor space using CTF tools (VSM Medtech Inc., Vancouver, Canada) and the ELAN software package developed in the Brain Dynamics and Cognition team (Lyon Neuroscience Research Center $^{1}$; Aguera et al., 2011). Source reconstruction and DCM analyses were performed with SPM8 (Wellcome Trust Centre for Neuroimaging, London, UK; Litvak et al., 2011) using MATLAB 7.6 (Mathworks Inc., Natick, MA, USA).

Individual MEG trials were automatically inspected from $-100 \mathrm{~ms}$ to $5500 \mathrm{~ms}$ with respect to the onset of the first S1 tone (i.e., a time window covering S1, the delay, S2 and an additional $500 \mathrm{~ms}$ after S2). Trials with ranges of values exceeding \pm 3000 fT within a $1000 \mathrm{~ms}$ sliding time-window at any sensor site

${ }^{1}$ http://elan.lyon.inserm.fr/
$( \pm 100 \mu \mathrm{V}$ at EOG channels) were excluded from the analysis: as a result, between 90 and 165 trials were kept for each participant and condition. After artifact rejection, two different secondorder Butterworth filters ( $12 \mathrm{~dB} /$ octave slope) were performed: (1) for transient evoked responses, a band-pass filter between 2 and $30 \mathrm{~Hz}$; and (2) for the analysis of the change-specific responses, filtering was done with a band-pass filter between 0.5 and $30 \mathrm{~Hz}$.

The analyses reported here focused on ERFs evoked by (1) the short-term memory retrieval of "Same" trials, corresponding to the average of tones 2-6 of the second melody of the pair (see procedure and rationale in Albouy et al., 2013a); (2) the changed tone in S2 for correct responses (i.e., during the detection of the changed tone) (Figure 1C). Note that because control participants had more correct responses than amusic participants, we used for each control participant the same number of trials as his/her matched amusic participant (selected randomly from the entire set of correct response trials). To analyze event-related responses following the "changed" tone in S2, two averages were performed for each participant (note that we always kept the baseline in the -100 to $0 \mathrm{~ms}$ interval before S1): firstly, an average of all correctly detected changed tones (in position $2-5$ ), in a -100 to $700 \mathrm{~ms}$ time-window around the onset of the change (this ERF 
thus combined data for differences in all possible positions in S2), and secondly, an average of tones from correctly classified "Same" trials with, for each participant, the same number of tones in position 2, 3, 4, or 5 as used for the ERFs of the "Different" trials.

For controls only, the change-specific response was also assessed by comparing "Different" and "Same" trials (using the equivalent (unchanged) tone (in the same position of the melody as the changed tone of the "Different" trial)) with DCM (for correct responses). As DCM attempts to explain differences between waveforms in terms of coupling changes among sources, we considered that, in amusics, the waveforms for "Same" and "Different" trials were too similar (see Figure 1C) to allow comparing them with a DCM analysis.

\section{DYNAMIC CAUSAL MODELING}

We used DCM as implemented in SPM8 (David and Friston, 2003; David et al., 2006; Garrido et al., 2007; Litvak et al., 2011). DCM uses the concept of effective connectivity, which refers explicitly to the influence that one neuronal system exerts over another. DCM models interactions among cortical regions and allows making inferences about system parameters and the influence of experimental factors on these parameters. This analysis method uses neural mass models (David and Friston, 2003) to explain source activity in terms of the ensemble dynamics of interacting inhibitory and excitatory subpopulations of neurons (Jansen and Rit, 1995). It emulates the activity of a cortical source using three neural subpopulations, each assigned to one of three cortical layers: (1) an excitatory subpopulation in the granular layer; (2) an inhibitory subpopulation in the supra-granular layer; and (3) a population of deep pyramidal cells in the infra-granular layer. In this model, the excitatory pyramidal cells receive excitatory and inhibitory inputs from local inter-neurons (via intrinsic connections, confined to the cortical sheet), and send excitatory outputs to remote cortical areas via extrinsic connections. Bottom-up connections (also referred to as forward connections) originate in the infra-granular layers and terminate in the granular layer. In contrast, top-down connections (also referred to as backward connections) link agranular layers and lateral connections originate in infra-granular layers and target all layers. Additionally, the model considers that all extrinsic cortico-cortical connections are excitatory and are mediated through the axons of pyramidal cells. Exogenous inputs to the model have the same characteristics as forward connections. By adopting this network architecture, DCM is able to assess how a given experimental manipulation activates a cortical pathway rather than a cortical area or source. This approach thus uses a biologically informed model that allows for inferences about the underlying neuronal networks generating evoked responses such as Event Related Potentials (ERPs) and ERFs.

Three different DCM analyses were performed: (1) the first analysis aimed at testing whether amusics' altered brain responses during short-term memory retrieval of "Same" trials (without changed tone) could be explained by changes in effective connectivity between bilateral auditory cortices and bilateral IFG, as well as within and between the two auditory cortices; (2) the second analysis investigated what kind of connectivity patterns support the detection of a deviant tone in the typically functioning brain. We compared and modeled in controls (grand average data of the controls) the difference in effective connectivity between "Different" and "Same" trials (for correct responses); (3) the third analysis aimed at testing whether amusics' altered brain responses during the detection of the changed tone ("Difference Wave") could be explained by changes in effective connectivity between sources of the same network. We aimed at characterizing the two participant groups with a high signal-to-noise ratio and to investigate the putative differences in effective connectivity between them. DCM analyses were thus applied to the grand average data at the sensor level. This is equivalent to a meta-subject analysis (corresponding to the average of nine participants per group). It is relevant to note that this approach comes with an anatomo-functional approximation in the sense that it disregards the inter-subject variability within each group. However, this approach makes it possible to test, with DCM, alternative mechanistic hypothesis about the network architecture and modulations in effective connectivity that differ (1) between amusic and control participants (analyses 2 and 3); and (2) between two conditions within a group (analysis 2). At the expense of assuming a meta-subject for each group, this approach provides quantitative conclusions at the population level that benefit from a higher signal-to-noise ratio and hence a greater sensitivity. We thus compared and modeled the difference between the grand average data of the controls (average over the nine participants) and the grand average data of the amusics (average over the nine participants at the sensor level) (as in Albouy et al., 2013a) and the model comparisons were thus based on Fixed effects.

For analysis (1) we modeled the data during the post-stimulus period 0-250 ms (corresponding to the inter-tone interval in melodies (see Albouy et al., 2013a). For analyses (2) and (3), we modeled the data during the post-stimulus period 0-700 ms. This latter period encompasses components of the ERFs that are assumed to reflect the detection of the changed tone (see Figures 1C, 2). Indeed, as described in Albouy et al. (2013a) and below, the processing of the changed tone in controls was associated with two evoked responses. The first evoked response was elicited approximately $150 \mathrm{~ms}$ after the onset of the changed tone; and the second one peaked at $500 \mathrm{~ms}$ after the onset of the changed tone.

\section{NETWORK MODEL SPECIFICATION AND BAYESIAN MODEL SELECTION}

All compared models were based on the same network architecture, which was motivated by (1) the results of our classical source reconstruction analysis of the brain responses evoked by the changed tone, which were revealing sources in a bilateral fronto-temporal network (see Table 2 and Albouy et al., 2013a for ROIs that were significantly different from baseline); and (2) the hypothesis of impaired fronto-temporal connectivity and inter-hemispheric connectivity, which were observed in congenital amusia with functional (Hyde et al., 2011), effective (Albouy et al., 2013a) and anatomical connectivity approaches (Loui et al., 2009). We assumed four sources, modeled as equivalent current dipoles (ECDs), over left and right primary auditory cortices (A1), left and right pars opercularis of the IFG 


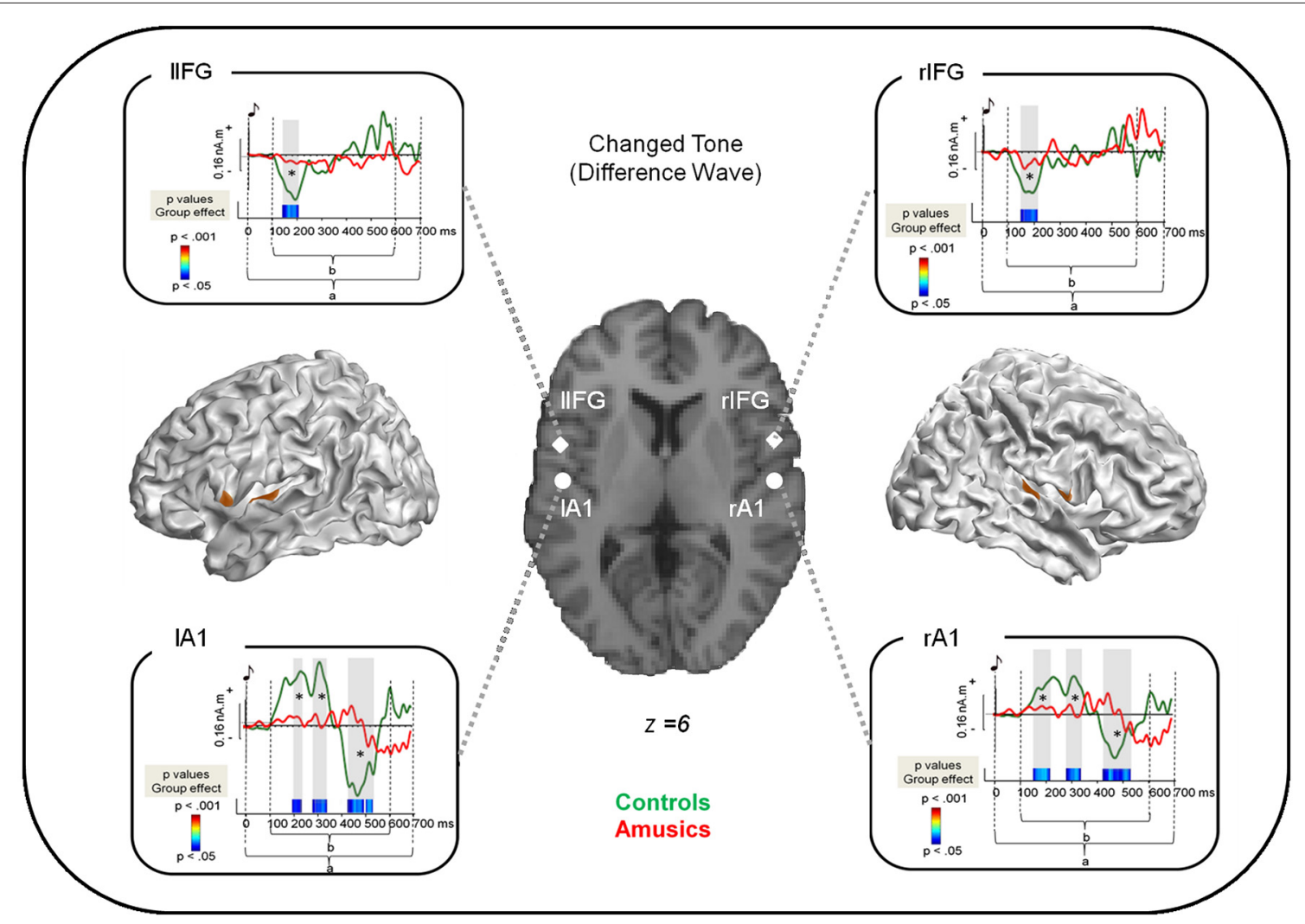

FIGURE 2 | Source reconstruction of the brain responses specifically evoked by the changed tone in S2. Cortical meshes show bilateral regions that were significantly different from baseline (as indicated by the brown areas). Coordinates of the peaks of activations are displayed on the single subject T1 image provided by SPM8 for four regions: the bilateral auditory cortices as well as the bilateral pars opercularis of the Inferior Frontal Gyrus (see Table 2). The surrounding panels correspond to the grand average of source data for each region and for the time window where the inversion was performed (0-700 ms after the changed tone onset, as indicated by a) for the control group (green) and the amusic group (red). Two sample $t$-tests were performed at each time sample and for each region on source amplitude in the 100-600 ms time window (as indicated by b) in the two groups of participants. $p$-values are reported across time below the source amplitudes with blue for $p<0.05$; green for $p<0.01$; and red for $p<0.001$. Note that only effects lasting longer than 15 ms were reported. See Albouy et al., 2013a for details. (see Table 2 and Figure 2). Using these sources, we constructed the following DCM (as in Albouy et al., 2013a): An extrinsic input entered bilaterally to the primary auditory cortices (A1), which were connected to their ipsilateral IFG. Inter-hemispheric (lateral) connections were placed between left and right A1. All connections were reciprocal (i.e., connected with forward and backward connections or with bilateral connections, see Figure 3). Given this network architecture, we used a (four) factorial design and performed family-level inference to assess modulations of effective connectivity underlying (1) the group difference in auditory evoked responses (analyses 1 and 3); and (2) the differences between "Different" and "Same" trials in controls (analysis 2). In all analyses, the first Factor pertains to the modulation of intrinsic connectivity in bilateral auditory cortices (and includes two families (or levels), corresponding to models where these intrinsic connections were modulated (or not) between the two groups/conditions). The second Factor pertains to the modulation of lateral connections between the two auditory cortices (two families: models that include a modulation or not). The third Factor relates to the type of connections between auditory and frontal areas that are modulated (that is, forward, backward, or both forward and backward connections) or not (resulting in four families). Finally, the fourth Factor pertains to the hemispheric location of the above described modulated connections, either in the right hemisphere, the left hemisphere, or both (resulting in three families). We thus fitted and compared 48 models for each analysis. Assuming uniform prior probabilities over families, we used Bayesian model selection (BMS) to compare them in fixed effects analysis (FFX; Penny et al., 2010). This rests upon the free energy (or approximate marginal likelihood or evidence) for each model, and yields a posterior probability associated with each model family.

\section{RESULTS}

Behavioral and MEG results of the present experiment were reported in detail in Albouy et al. (2013a; p. 1646-1655). This result section presents a short summary of the behavioral and MEG results to support the comprehension of the new DCM analyses performed on the data. 
Table 2 | Frontal and temporal generators of the change-specific response within S2.

\begin{tabular}{|c|c|c|c|c|c|c|c|}
\hline Lobe & Region & Hemisphere & $x$ & $y$ & $z$ & $\mathrm{~mm}^{2}$ & nb \\
\hline Frontal & & L & -54 & 3 & 6 & 65 & 12 \\
\hline & & L & -52 & -12 & 5 & 117 & 16 \\
\hline
\end{tabular}

Coordinates correspond to the vertex with maximal amplitude within each region (coordinates are in MNI space). IFG, Inferior Frontal Gyrus; STG, Superior Temporal Gyrus; PT, Planum Temporale; nb, number of vertices.

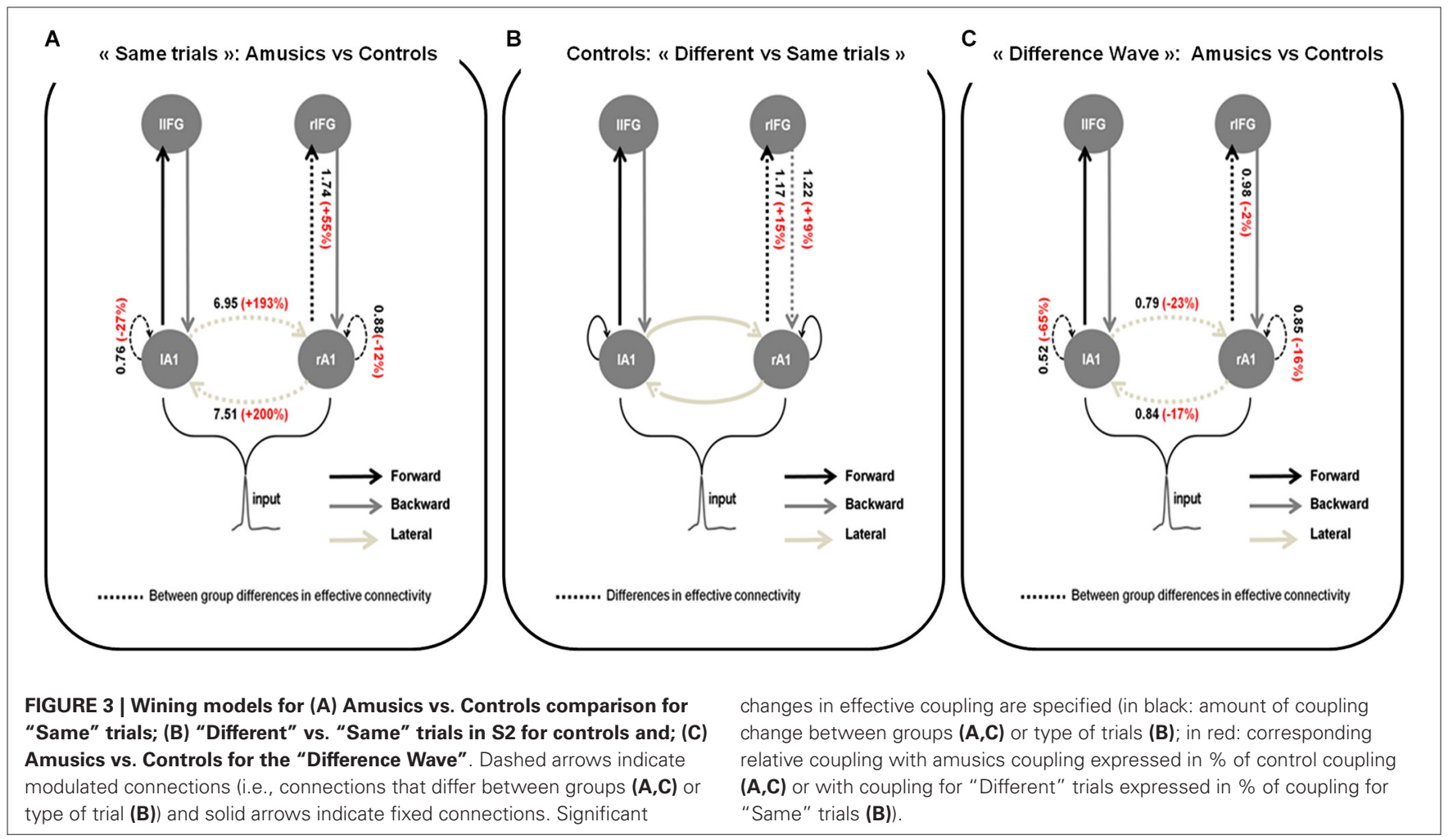

\section{BEHAVIORAL DATA}

Performance was significantly above chance (i.e., 0\% of HitsFalse Alarms (FAs)) in each group ( $t$-tests, all ps<0.0001). Hits-FAs were analyzed with a one-way ANOVA and revealed that the main effect of Group $\left(F_{(1,16)}=103.83 ; p<0.0001\right.$; $\left.M S E=88.39 ; \eta_{p}^{2}=0.86\right)$, was significant: all amusic participants exhibited a deficit in the melodic contour task in comparison to controls (Figure 1B).

To investigate participants' overall strategy (missing pitch changes or hearing non-existing changes), additional analyses were performed by investigating the effect of Trial Type (same, different) in participants' performance. We analyzed the percentage of correct responses with a $2 \times 2$ ANOVA, with Group as between-participants factor and Trial Type (same, different) as within-participant factors. In addition to the main effect of Group $\left(F_{(1,16)}=107.06 ; p<0.0001 ; M S E=43.0 ; \eta_{p}^{2}=0.86\right)$, the effect of Trial Type was significant $\left(F_{(1,16)}=49.97 ; p<0.0001\right.$; $\left.M S E=73.4 ; \eta_{p}^{2}=0.75\right)$ revealing that participants' performance was decreased for "Different" trials in comparison to "Same" trials. Finally, the Group-by-Trial-Type interaction was significant $\left(F_{(1,16)}=28.37 ; p<0.0001 ; M S E=73.4 ; \eta_{p}^{2}=0.63\right)$. To analyze this interaction, Fischer LSD post hoc tests were carried out and revealed that while controls show similar performance for "Same" and "Different" trials $(p=0.23)$ amusics show decreased performance for "Different" trials as compared to "Same" trials $(p<0.001)$. Note that amusic performance was decreased as compared to controls for both "Same" ( $p=0.048)$ and "Different" trials $(p<0.0001)$.

\section{MEG RESULTS}

Source analyses of the transient responses of the changed tone in S2

We summarize here the analyses presented in Albouy et al. (2013a) concerning the brain responses evoked by the changed tone in the S2 melody of "Different" trials (for correct responses). The "Difference Wave" ("Different" trials minus "Same" trials) observed at the sensor level revealed that for controls, the processing of the changed tone was associated with two evoked 
responses: The first one was elicited approximately $150 \mathrm{~ms}$ after the onset of the changed tone; and the second one peaked at 500 ms after the tone onset. This biphasic response was altered in amusics (Figure 1C, right panel).

Source modeling of the "Difference Wave" (i.e., between "Different" trials and "Same" trials for correct responses, see Section Methods) revealed that activity was significantly different from baseline in bilateral fronto-temporal regions (see Table 2).

Two-sample $t$-tests (corrected for multiple comparisons, see Albouy et al., 2013a) revealed group differences in source amplitude, with higher amplitudes for control than for amusic participants in the four regions of interest and in the following time windows: (1) Right STG, 150-210 ms; 290-340 ms; 425-520 ms; (2) rIFG, 160-210 ms; (3) Left IFG (IIFG), 150-205 ms, and; (4) Left STG, 190-230 ms; 280-340 ms; 425-530 ms (Figure 2).

\section{DYNAMIC CAUSAL MODELING}

For each DCM analysis, the four family-wise inference allowed us to retained a winning model (see Figure 3 ) based on posterior probabilities (criterion of $p>0.99$ ).

\section{Group comparison for S2 in "Same" trials}

Figure 3A shows the network architecture of the winning model as well as the conditional estimates of the connection strengths associated with the connections that were significantly modulated to explain the amusic response compared to the control response for S2 in "Same" trials. Posterior estimates obtained with the winning model enabled us to conclude that for S2 in "Same" trials, compared to controls, amusic participants showed an abnormally increased lateral connectivity between the two auditory cortices, decreased intrinsic modulations in both auditory cortices, and increased forward connectivity from the right auditory cortex to the right IFG.

\section{Comparison between "Different" and "Same" trials for controls}

In control participants (Figure 3B), increased right lateralized temporo-frontal connections (both Forward and Backward) best explain the brain responses to "Different" trials as compared to "Same" trials. We found strong evidence in favor of this lateralized network compared with the set of alternative hypotheses, with a high posterior model probability $>0.99$.

\section{Group comparison for the "Difference Wave"}

When investigating the "Difference Wave", posterior estimates enabled us to conclude that, amusic participants, in comparison to controls, showed decreased lateral connectivity between the two auditory cortices, decreased intrinsic modulations in both auditory cortices, and decreased forward connectivity between the right auditory cortex and the right IFG (see Figure 3C).

\section{Lateralization of the effects}

Finally, to investigate the putative imbalance of the observed effects, between the right and left hemispheres, we investigated whether group differences reported above in terms of relative coupling (with amusics coupling expressed in \% of control coupling) differed between the right and left auditory cortices.
Therefore we compared with DCM (for the wining models of analyses 1 and 3) each amusic participant to his/her matched control participant. When comparing the modulation values of intrinsic connections with a paired $t$-test, between the right and left auditory cortices for "Same" trials in S2 (analysis 1) and for the "Difference Wave" (analysis 3), no significant difference was observed (all ps $>0.19$ ).

\section{DISCUSSION}

The present study aimed at extending the characterization of cerebral correlates of the short-term memory deficit for pitch in congenital amusia. The data revealed that in addition to the functional abnormalities observed in the right fronto-temporal pathway during encoding of pitch information (Albouy et al., 2013a), amusics' pitch deficits are also associated with an altered functioning of the same network (bilateral IFG and auditory cortex) during the short-term memory retrieval of melodies. To explain these group differences in terms of differences of coupling among sources, we performed group comparisons with DCM. These analyses revealed that in comparison to controls, the amusic brain is characterized by abnormal connections between and within the two auditory cortices as well as abnormal right forward temporal-to-frontal effective connectivity during shortterm memory retrieval. These findings, along with additional analyses in controls, suggest that the short-term memory retrieval of melodic information and the detection of a deviant/unexpected tone in the normally functioning brain (leading to a prediction error, (Bastos et al., 2012; Dietz et al., 2014; Friston et al., 2014)) are supported by temporo-frontal interactions.

\section{ALTERED SHORT-TERM RETRIEVAL OF MELODIC INFORMATION IN CONGENITAL AMUSIA}

As reported in Albouy et al. (2013a), source reconstruction of the ERFs elicited by the changed tone (the "Difference Wave" between correct "Different" trials and "Same" trials) allowed us to observe activity in bilateral auditory cortices and bilateral pars opercularis of the IFG (BA 44) in the control group. This is in line with previous research suggesting that the short-term memory retrieval of pitch information involves (1) brain areas that support the perceptual representation of that information (Owen, 2000; D'Esposito, 2007; Grimault et al., 2014), and (2) areas of the Ventro-lateral Prefrontal Cortex (IFG), that has been described as supporting low-level mnemonic processes, such as rehearsal and retrieval in short-term recognition (Owen, 2000). In contrast, the amusic brain exhibited an abnormal recruitment of these regions for pitch retrieval. These data thus further reflect the functional correlates of the short-term memory deficit in congenital amusia (see Albouy et al., 2013a).

Most interestingly, while encoding and short-term memory retrieval of melodic information recruit similar brain regions, the present data showed that the connectivity patterns between these regions can differ between those two stages.

\section{ALTERED INTRINSIC AND LATERAL CONNECTIVITY IN THE AUDITORY CORTEX OF THE AMUSIC BRAIN}

During the short-term memory retrieval of pitch information, for both "Same" trials and for the "Difference Wave", the amusic 
brain showed abnormally reduced intrinsic connections within the bilateral auditory cortices in comparison to controls. This finding supports the fact that while amusics' auditory cortex seems to present near normal functioning in response to pitch information in a simple context (passive or active listening without memory) (Peretz et al., 2005, 2009; Moreau et al., 2009, 2013; Hyde et al., 2011; Peretz, 2013), it elicits abnormal responses when higher-level processing is required (Albouy et al., 2013a). Interestingly, reduced intrinsic connectivity within both auditory cortices in the amusic brain has been observed also during the encoding of pitch information (see Albouy et al., 2013a), thus suggesting that primary sensory areas are recruited during both encoding and retrieval in a short-term memory task (Zatorre et al., 1994; Griffiths, 1999; Griffiths et al., 1999; Owen, 2000; Curtis and D'Esposito, 2003; Gaab et al., 2003; Peretz and Zatorre, 2005; D'Esposito, 2007; Logie and D'Esposito, 2007; Schulze et al., 2009). Moreover, this hypothesis is in line with studies suggesting that the detection of deviant tones (Garrido et al., 2007, 2008, 2009a,b; Lieder et al., 2013) in an oddball paradigm (Mismatch Negativity, MMN; Näätänen, 1992) is also supported by intrinsic connections within the auditory cortex, as revealed by DCM analyses. However, it should be noted that in the present study, the comparison between "Different" and "Same" trials in controls, did not specifically show the implication of these intrinsic connections for the detection of pitch deviance. Based on the present data, we can only propose that modulations of intrinsic connections are reduced in congenital amusics in comparison to controls not only during encoding, but also during short-term memory retrieval of pitch information.

In addition to the altered functioning of auditory cortices during short-term memory retrieval of pitch information, the analysis of the "Difference Wave" revealed reduced lateral connection strengths between the right and left auditory cortices in amusics in comparison to controls. This result contrasts with the group comparison performed for S2 in "Same" trials (see above, Figure 3A) and with the abnormal connectivity pattern in the amusic brain reported for pitch perception, notably during both passive listening (Hyde et al., 2011) and active encoding in a short-term memory task (Albouy et al., 2013a). There, hyper-connectivity between the two auditory cortices has been observed in amusics as compared to controls. This abnormal hyper-connectivity between the two auditory cortices in the amusic brain might be a marker of the pitch processing deficit, as also observed in other developmental disorders (see Wolf et al., 2010) for dyslexia; and see (Hyde et al., 2011) for converging evidence in amusia) or rather reveal compensatory mechanisms of the amusic brain. This would suggest that amusics might compensate for an impoverished processing in the right auditory cortex by recruiting the contralateral auditory cortex. Note that for the group comparison performed on the "Difference Wave" (Figure 3C), we removed brain responses related to the encoding of the information of S2 (for which increased lateral connectivity is observed in amusics), by computing the difference between brain responses in "Different" trials and in "Same" trials. The ERFs studied here can thus be considered as being specific to the detection of the deviance in the "Different" trials (and thus, the difference between the information retrieved from memory and the presented tone). Results thus suggest that lateral connections have a role in detecting an auditory event that mismatches with a memory trace of a previously heard/memorized stimulus (here S1). However, note that the specific role of these lateral connections in pitch short-term memory retrieval was not demonstrated in the typically functioning brain (data of controls) in the present study ("Different" vs. "Same" trials in controls).

\section{ALTERED FORWARD VS. BACKWARD CONNECTIVITY BETWEEN THE rA1 AND rIFG}

The DCM analyses revealed abnormal connectivity in the amusic brain in a right fronto-temporal network during the short-term memory retrieval of melodic information (for both the processing of "Same" trials and the processing of the changed tone). This right-lateralized abnormality is in line with previous research showing functional and anatomical alteration of this pathway in congenital amusia (Loui et al., 2009; Hyde et al., 2011; Albouy et al., 2013a; Lévêque et al., submitted). Moreover, the present data further support the hypothesis that the processing of pitch information in the brain is, to some extent, asymmetric with a right-hemispheric predominance (with both hemispheres being involved) as it has been previously suggested based on musical deficits observed for patients with right-hemispheric brain lesions (Zatorre and Samson, 1991; Peretz, 1996, 2001; Peretz et al., 1997; Patel et al., 1998; Steinke et al., 2001; Nicholson et al., 2002, 2003; Stewart et al., 2006), and by neuroimaging studies in typical listeners (Zatorre et al., 1994, 2002; Griffiths, 1999; Griffiths et al., 1999; Janata et al., 2002a,b; Tillmann et al., 2003, 2006; Koelsch et al., 2005, 2009; Peretz and Zatorre, 2005; Hyde et al., 2008; Stewart et al., 2008; Schulze et al., 2009, 2011a,b).

However, while an abnormal frontal-to-temporal backward connectivity in the right hemisphere has been observed during the encoding of pitch information in the amusic brain (see Albouy et al., 2013a), the present analyses revealed that during shortterm memory retrieval (for "Same" trials and the "Difference Wave"), amusics exhibited an abnormal temporal-to-frontal forward connectivity between the right auditory cortex and the right IFG in comparison to controls. According to Garrido et al. (2007), auditory evoked brain responses are mediated by interactions between fronto-temporal cortical areas. This differential role of forward and backward connections in auditory perception can be interpreted within the predictive coding framework (Bastos et al., 2012; Dietz et al., 2014; Friston et al., 2014).

According to predictive coding principles, neural systems are able to predict statistical regularities in the environment based on prior experience. In this view, neural systems can attenuate responses to predictable events (Lecaignard et al., submitted), thanks to top-down predictions (conveyed by backward connections (Friston, 2003)) and the minimization of deviations from these predictions (i.e., the minimization of prediction errors). In contrast, for unpredictable events, bottomup prediction error signals (supported by forward connections (Penny et al., 2004)) emerge and report the "newsworthy" 
information from a lower hierarchical level (sensory input) that was not predicted by the higher level (prediction, memory trace of the prior experience). Along these lines, a prediction error can emerge due to either an inefficient construction of the prediction (no prediction or incorrect prediction) or to the occurrence of unpredictable events.

In the short-term memory paradigm used in the present study, it might be hypothesized that during short-term memory retrieval for "Same" trials, in the typically functioning brain, the neural system may minimize prediction error signals when the prior information (here S1) has been well encoded and the developed predictions (appropriate predictions) are fulfilled in the S2 melody ("Same" trials). In contrast, the perception of "Different" trials (that mismatches with predictions for one tone) may generate prediction errors signals. This hypothesis has received support from the DCM analysis performed in the control group (analysis 2), which showed increased forward effective connectivity between the right auditory cortex and the right IFG in "Different" trials as compared to "Same" trials. Moreover and interestingly, this increased temporo-frontal connectivity was associated with increased backward frontal-totemporal connectivity that could be considered as an update of the prediction model after the reception of the prediction error signal.

In the amusic brain, however, it can be also hypothesized that disruptions in predictive coding can underlie the abnormal percepts. In Albouy et al. (2013a), altered backward connectivity during the encoding of melodic contour patterns in the amusic brain has been reported. According to the predictive coding principles described above, this result could be interpreted as follows: the neural system needs to keep a trace of a previously presented stimulus in memory (top-down processes) while processing a new stimulus arriving in the perceptual system (Demany and Semal, 2008). This is done in order to build an appropriate memory trace (and prediction) of the incoming melodic pattern. Observing decreased effective backward connectivity from the right IFG to the right auditory cortex in the amusic brain could be interpreted as reflecting a decreased or vague prediction (relying on top-down backward connections). By considering that the memory trace (as the basis for the prediction) has not been appropriately constructed (altered encoding), the amusic brain might have build incorrect prediction, and inappropriate prediction errors signals can thus emerge during short-term memory retrieval, and thus even for "Same" trials.

Interestingly, in the present study, we observed increased forward connectivity between the right auditory cortex and the right IFG in the amusic brain, as compared to controls, for the processing of S2 in "Same" trials. Along these lines it might thus be hypothesized that as the first melody of the pair has not been well encoded (see Albouy et al., 2013a), tones occurring in the perceptual system during short-term retrieval (S2) could be considered as deviant tones by the amusic brain. It should be considered however that these prediction errors signals should occur similarly for "Same" and "Different" trials as every event of S2 can be considered as unexpected (not or weakly predicted) by the amusic brain. This notably finds support in the group comparison for the "Difference Wave" (analysis 3) for which only a small difference in the forward connection (that conveys prediction error signal) was observed between amusics and controls (2\%). This suggests that in both groups, the right auditory cortex is sending prediction error signals when the deviant tone occurs, but the mechanisms causing these signals might differ between the two groups (incorrect prediction in amusics, detection of unpredictable event in controls as shown by the comparison of "Same" and "Different" trials, analysis 2).

Overall, the present study showed that in the amusic brain, forward temporal-to-frontal connectivity seems to be altered during short-term memory retrieval of melodies, thus comforting the hypothesis of impaired functioning of the right frontotemporal network in this developmental disorder (Loui et al., 2009; Hyde et al., 2011; Albouy et al., 2013a; Peretz, 2013; Tillmann et al., 2015). This result is of interest because it suggests that frontal and temporal cortices support both encoding and short-term memory retrieval of melodic information, but their interaction is modulated in different directions for the two processing steps related to memory.

\section{CONCLUSION}

In congenital amusia, an altered functioning of a bilateral frontotemporal network during the short-term memory retrieval of melodic information was associated with altered connectivity patterns in a right fronto-temporal network as well as within and between the two auditory cortices. These data improve our understanding about the role of frontal and temporal structures (including auditory cortices) and of the frontotemporal pathway in music processing, as well as its impairment in this developmental disorder. To pursue the characterization of the deficits in congenital amusia, further research investigating these encoding and retrieval mechanisms for other types of auditory information (such as verbal material) as well as for other modalities (such as visual memory) are needed. This will allow determining whether connectivity patterns for visual memory (Sneve et al., 2013) are preserved or not in amusia, and if the altered brain responses and connectivity patterns observed in the amusic brain for tone sequences could be considered as a general impairment of auditory processing or are specific for pitch.

\section{AUTHOR CONTRIBUTIONS}

Philippe Albouy, Barbara Tillmann and Anne Caclin designed the experiment, created the auditory stimuli, and programmed the tasks. Philippe Albouy, Barbara Tillmann, and Anne Caclin collected the data. Philippe Albouy analyzed the data. Jérémie Mattout and Gaëtan Sanchez provided the routines for the DCM analyses and supervised data analyses. Philippe Albouy, Jérémie Mattout, Gaëtan Sanchez, Barbara Tillmann and Anne Caclin interpreted the results and Philippe Albouy wrote the first draft of the article. All authors critically revised the article and approved the final version. The authors are accountable for all aspects of the work and ensure that questions related to the accuracy or integrity of any part of the work are appropriately investigated and resolved. 


\section{ACKNOWLEDGMENTS}

This work was supported by a grant from the Rhône Alpes Cluster $n^{\circ}$ 11: "Handicap, vieillissement, neurosciences" to Barbara Tillmann and by a grant from "Agence Nationale de la Recherche" (ANR) of the French Ministry of Research ANR-11-BSH2-00101 to Barbara Tillmann and Anne Caclin. Philippe Albouy was funded by a PhD fellowship of the CNRS. Gaëtan Sanchez was funded by the French ANR project ANR-DEFIS 09-EMER-002 CoAdapt to Jérémie Mattout. This work was conducted in the framework of the LabEx CeLyA ("Centre Lyonnais d'Acoustique", ANR-10-LABX-0060) and of the LabEx Cortex ("Construction, Function and Cognitive Function and Rehabilitation of the Cortex", ANR-11-LABX-0042) of Université de Lyon, within the program "Investissements d'avenir" (ANR-11-IDEX-0007) operated by the French National Research Agency (ANR). We thank Dolly-Anne Muret for her collaboration in the construction of the experimental material.

\section{REFERENCES}

Aguera, P. E., Jerbi, K., Caclin, A., and Bertrand, O. (2011). ELAN: a software package for analysis and visualization of MEG, EEG and LFP signals. Comput. Intell. Neurosci. 2011:158970. doi: 10.1155/2011/158970

Albouy, P., Mattout, J., Bouet, R., Maby, E., Sanchez, G., Aguera, P. E., et al. (2013a). Impaired pitch perception and memory in congenital amusia: the deficit starts in the auditory cortex. Brain 136, 1639-1661. doi: 10.1093/brain/ awt082

Albouy, P., Schulze, K., Caclin, A., and Tillmann, B. (2013b). Does tonality boost short-term memory in congenital amusia? Brain Res. 1537, 224-232. doi: 10. 1016/j.brainres.2013.09.003

Ayotte, J., Peretz, I., and Hyde, K. (2002). Congenital amusia: a group study of adults afflicted with a music-specific disorder. Brain 125, 238-251. doi: 10. 1093/brain/awf028

Bastos, A. M., Usrey, W. M., Adams, R. A., Mangun, G. R., Fries, P., and Friston, K. J. (2012). Canonical microcircuits for predictive coding. Neuron 76, 695-711. doi: 10.1016/j.neuron.2012.10.038

Curtis, C. E., and D'Esposito, M. (2003). Persistent activity in the prefrontal cortex during working memory. Trends Cogn. Sci. 7, 415-423. doi: 10.1016/s13646613(03)00197-9

David, O., and Friston, K. J. (2003). A neural mass model for MEG/EEG: coupling and neuronal dynamics. Neuroimage 20, 1743-1755. doi: 10.1016/j.neuroimage. 2003.07.015

David, O., Kiebel, S. J., Harrison, L. M., Mattout, J., Kilner, J. M., and Friston, K. J. (2006). Dynamic causal modeling of evoked responses in EEG and MEG. Neuroimage 30, 1255-1272. doi: 10.1016/j.neuroimage.2005.10.045

Demany, L., and Semal, C. (2008). "The role of memory in auditory perception," in Auditory Perception of Sound Sources, eds W. A. Yost, A. N. Popper and R. R. Fay (Springer: Springer Handbook of Auditory Research), 77-113.

D'Esposito, M. (2007). From cognitive to neural models of working memory. Philos. Trans. R. Soc. Lond. B Biol. Sci. 362, 761-772. doi: 10.1098/rstb.2007. 2086

Dietz, M. J., Friston, K. J., Mattingley, J. B., Roepstorff, A., and Garrido, M. I. (2014). Effective connectivity reveals right-hemisphere dominance in audiospatial perception: implications for models of spatial neglect. J. Neurosci. 34, 50035011. doi: 10.1523/JNEUROSCI.3765-13.2014

Foster, N. E., Halpern, A. R., and Zatorre, R. J. (2013). Common parietal activation in musical mental transformations across pitch and time. Neuroimage 75, 27-35. doi: 10.1016/j.neuroimage.2013.02.044

Foster, N. E., and Zatorre, R. J. (2010). A role for the intraparietal sulcus in transforming musical pitch information. Cereb. Cortex 20, 1350-1359. doi: 10. 1093/cercor/bhp199

Foxton, J. M., Dean, J. L., Gee, R., Peretz, I., and Griffiths, T. D. (2004). Characterization of deficits in pitch perception underlying "tone deafness". Brain 127, 801-810. doi: 10.1093/brain/awh105

Friston, K. (2003). Learning and inference in the brain. Neural Netw. 16, 1325-1352. doi: 10.1016/j.neunet.2003.06.005
Friston, K. J., Bastos, A. M., Pinotsis, D., and Litvak, V. (2014). LFP and oscillationswhat do they tell us? Curr. Opin. Neurobiol. 31C, 1-6. doi: 10.1016/j.conb.2014. 05.004

Friston, K. J., Harrison, L., and Penny, W. (2003). Dynamic causal modelling. Neuroimage 19, 1273-1302. doi: 10.1016/s1053-8119(03)00202-7

Gaab, N., Gaser, C., Zaehle, T., Jancke, L., and Schlaug, G. (2003). Functional anatomy of pitch memory-an fMRI study with sparse temporal sampling. Neuroimage 19, 1417-1426. doi: 10.1016/s1053-8119(03)00224-6

Garrido, M. I., Friston, K. J., Kiebel, S. J., Stephan, K. E., Baldeweg, T., and Kilner, J. M. (2008). The functional anatomy of the MMN: a DCM study of the roving paradigm. Neuroimage 42, 936-944. doi: 10.1016/j.neuroimage.2008. 05.018

Garrido, M. I., Kilner, J. M., Kiebel, S. J., and Friston, K. J. (2007). Evoked brain responses are generated by feedback loops. Proc. Natl. Acad. Sci. U S A 104, 20961-20966. doi: 10.1073/pnas.0706274105

Garrido, M. I., Kilner, J. M., Kiebel, S. J., and Friston, K. J. (2009a). Dynamic causal modeling of the response to frequency deviants. J. Neurophysiol. 101, 2620-2631. doi: 10.1152/jn.90291.2008

Garrido, M. I., Kilner, J. M., Stephan, K. E., and Friston, K. J. (2009b). The mismatch negativity: a review of underlying mechanisms. Clin. Neurophysiol. 120, 453-463. doi: 10.1016/j.clinph.2008.11.029

Gosselin, N., Jolicoeur, P., and Peretz, I. (2009). Impaired memory for pitch in congenital amusia. Ann. N Y Acad. Sci. 1169, 270-272. doi: 10.1111/j.1749-6632. 2009.04762.x

Griffiths, T. D. (1999). Human complex sound analysis. Clin. Sci. (Lond). 96, 231234. doi: $10.1042 / \mathrm{cs} 19980337$

Griffiths, T. D., Johnsrude, I., Dean, J. L., and Green, G. G. (1999). A common neural substrate for the analysis of pitch and duration pattern in segmented sound? Neuroreport 10, 3825-3830. doi: 10.1097/00001756-199912160-00019

Grimault, S., Nolden, S., Lefebvre, C., Vachon, F., Hyde, K., Peretz, I., et al. (2014). Brain activity is related to individual differences in the number of items stored in auditory short-term memory for pitch: evidence from magnetoencephalography. Neuroimage 94, 96-106. doi: 10.1016/j.neuroimage. 2014.03.020

Hyde, K. L., Lerch, J. P., Zatorre, R. J., Griffiths, T. D., Evans, A. C., and Peretz, I. (2007). Cortical thickness in congenital amusia: when less is better than more. J. Neurosci. 27, 13028-13032. doi: 10.1523/jneurosci.3039-07.2007

Hyde, K. L., and Peretz, I. (2004). Brains that are out of tune but in time. Psychol. Sci. 15, 356-360. doi: 10.1111/j.0956-7976.2004.00683.x

Hyde, K. L., Peretz, I., and Zatorre, R. J. (2008). Evidence for the role of the right auditory cortex in fine pitch resolution. Neuropsychologia 46, 632-639. doi: 10. 1016/j.neuropsychologia.2007.09.004

Hyde, K. L., Zatorre, R. J., Griffiths, T. D., Lerch, J. P., and Peretz, I. (2006). Morphometry of the amusic brain: a two-site study. Brain 129, 2562-2570. doi: 10.1093/brain/awl204

Hyde, K. L., Zatorre, R. J., and Peretz, I. (2011). Functional MRI evidence of an abnormal neural network for pitch processing in congenital amusia. Cereb. Cortex 21, 292-299. doi: 10.1093/cercor/bhq094

Janata, P., Birk, J. L., Van Horn, J. D., Leman, M., Tillmann, B., and Bharucha, J. J. (2002a). The cortical topography of tonal structures underlying Western music. Science 298, 2167-2170. doi: 10.1126/science. 1076262

Janata, P., Tillmann, B., and Bharucha, J. J. (2002b). Listening to polyphonic music recruits domain-general attention and working memory circuits. Cogn. Affect. Behav. Neurosci. 2, 121-140. doi: 10.3758/cabn.2.2.121

Jansen, B. H., and Rit, V. G. (1995). Electroencephalogram and visual evoked potential generation in a mathematical model of coupled cortical columns. Biol. Cybern. 73, 357-366. doi: 10.1007/bf00199471

Jiang, C., Lim, V. K., Wang, H., and Hamm, J. P. (2013). Difficulties with pitch discrimination influences pitch memory performance: evidence from congenital amusia. PLoS One 8:e79216. doi: 10.1371/journal.pone.0079216

Jones, J. L., Zalewski, C., Brewer, C., Lucker, J., and Drayna, D. (2009). Widespread auditory deficits in tune deafness. Ear Hear. 30, 63-72. doi: 10.1097/AUD. 0b013e31818ff95e

Koelsch, S., Gunter, T. C., Wittfoth, M., and Sammler, D. (2005). Interaction between syntax processing in language and in music: an ERP study. J. Cogn. Neurosci. 17, 1565-1577. doi: 10.1162/089892905774597290

Koelsch, S., Schulze, K., Sammler, D., Fritz, T., Müller, K., and Gruber, O. (2009). Functional architecture of verbal and tonal working memory: an FMRI study. Hum. Brain Mapp. 30, 859-873. doi: 10.1002/hbm.20550 
Lieder, F., Stephan, K. E., Daunizeau, J., Garrido, M. I., and Friston, K. J. (2013). A neurocomputational model of the mismatch negativity. PLoS Comput. Biol. 9:e1003288. doi: 10.1371/journal.pcbi.1003288

Litvak, V., Mattout, J., Kiebel, S., Phillips, C., Henson, R., Kilner, J., et al. (2011). EEG and MEG data analysis in SPM8. Comput. Intell. Neurosci. 2011:852961. doi: $10.1155 / 2011 / 852961$

Liu, F., Patel, A. D., Fourcin, A., and Stewart, L. (2010). Intonation processing in congenital amusia: discrimination, identification and imitation. Brain 133, 1682-1693. doi: 10.1093/brain/awq089

Logie, R. H., and D’Esposito, M. (2007). Working memory in the brain. Cortex 43, 1-4. doi: 10.1016/S0010-9452(08)70441-6

Loui, P., Alsop, D., and Schlaug, G. (2009). Tone deafness: a new disconnection syndrome? J. Neurosci. 29, 10215-10220. doi: 10.1523/JNEUROSCI.1701-09. 2009

Maess, B., Koelsch, S., Gunter, T. C., and Friederici, A. D. (2001). Musical syntax is processed in Broca's area: an MEG study. Nat. Neurosci. 4, 540-545. doi: 10. $1038 / 87502$

Mandell, J., Schulze, K., and Schlaug, G. (2007). Congenital amusia: an auditorymotor feedback disorder? Restor. Neurol. Neurosci. 25, 323-334.

Moreau, P., Jolicoeur, P., and Peretz, I. (2009). Automatic brain responses to pitch changes in congenital amusia. Ann. N Y Acad. Sci. 1169, 191-194. doi: 10.1111/j. 1749-6632.2009.04775.x

Moreau, P., Jolicoeur, P., and Peretz, I. (2013). Pitch discrimination without awareness in congenital amusia: evidence from event-related potentials. Brain Cogn. 81, 337-344. doi: 10.1016/j.bandc.2013.01.004

Näätänen, R. (1992). Attention and Brain Function. Hillsdale, NJ: Erlbaum.

Nicholson, K. G., Baum, S., Cuddy, L. L., and Munhall, K. G. (2002). A case of impaired auditory and visual speech prosody perception after right hemisphere damage. Neurocase 8, 314-322. doi: 10.1093/neucas/8.4.314

Nicholson, K. G., Baum, S., Kilgour, A., Koh, C. K., Munhall, K. G., and Cuddy, L. L. (2003). Impaired processing of prosodic and musical patterns after right hemisphere damage. Brain Cogn. 52, 382-389. doi: 10.1016/s02782626(03)00182-9

Owen, A. M. (2000). The role of the lateral frontal cortex in mnemonic processing: the contribution of functional neuroimaging. Exp. Brain Res. 133, 33-43. doi: 10. 1007/978-3-642-59794-7_5

Patel, A. D., Foxton, J. M., and Griffiths, T. D. (2005). Musically tone-deaf individuals have difficulty discriminating intonation contours extracted from speech. Brain Cogn. 59, 310-313. doi: 10.1016/j.bandc.2004. 10.003

Patel, A. D., Peretz, I., Tramo, M., and Labreque, R. (1998). Processing prosodic and musical patterns: a neuropsychological investigation. Brain Lang. 61, 123-144. doi: 10.1006/brln.1997.1862

Patel, A. D., Wong, M., Foxton, J., Lochy, A., and Peretz, I. (2008). Speech Intonation perception deficits in musical tone deafness (congenital amusia). Music Percept. 25, 357-368. doi: 10.1525/mp.2008.25.4.357

Penny, W. D., Stephan, K. E., Daunizeau, J., Rosa, M. J., Friston, K. J., Schofield, T. M., et al. (2010). Comparing families of dynamic causal models. PLoS Comput. Biol. 6:e1000709. doi: 10.1371/journal.pcbi.1000709

Penny, W. D., Stephan, K. E., Mechelli, A., and Friston, K. J. (2004). Modelling functional integration: a comparison of structural equation and dynamic causal models. Neuroimage 23(Suppl. 1), S264-S274. doi: 10.1016/j.neuroimage.2004. 07.041

Peretz, I. (1996). Can we lose memory for music? A case of music agnosia in a nonmusician. J. Cogn. Neurosci. 8, 481-496. doi: 10.1162/jocn.1996.8. 6.481

Peretz, I. (2001). Brain specialization for music. New evidence from congenital amusia. Ann. N Y Acad. Sci. 930, 153-165. doi: 10.1111/j.1749-6632.2001. tb05731.x

Peretz, I. (2013). "The biological foundations of music: insights from congenital amusia," in The Psychology of Music, ed D. Deutsch (Amsterdam: Elsevier), 551564.

Peretz, I., Ayotte, J., Zatorre, R. J., Mehler, J., Ahad, P., Penhune, V. B., et al. (2002). Congenital amusia: a disorder of fine-grained pitch discrimination. Neuron 33, 185-191. doi: 10.1016/S0896-6273(01)00580-3

Peretz, I., Belleville, S., and Fontaine, S. (1997). Dissociations between music and language functions after cerebral resection: a new case of amusia without aphasia. Can. J. Exp. Psychol. 51, 354-368. doi: 10.1037/1196-1961.51. 4.354
Peretz, I., Brattico, E., Järvenpää, M., and Tervaniemi, M. (2009). The amusic brain: in tune, out of key and unaware. Brain 132, 1277-1286. doi: 10. 1093/brain/awp055

Peretz, I., Brattico, E., and Tervaniemi, M. (2005). Abnormal electrical brain responses to pitch in congenital amusia. Ann. Neurol. 58, 478-482. doi: 10. 1002/ana.20606

Peretz, I., Champod, A. S., and Hyde, K. (2003). Varieties of musical disorders. The montreal battery of evaluation of amusia.. Ann. N Y Acad. Sci. 999, 58-75. doi: 10.1196/annals.1284.006

Peretz, I., and Hyde, K. L. (2003). What is specific to music processing? Insights from congenital amusia. Trends Cogn. Sci. 7, 362-367. doi: 10.1016/s13646613(03)00150-5

Peretz, I., and Zatorre, R. J. (2005). Brain organization for music processing. Annu. Rev. Psychol. 56, 89-114. doi: 10.1146/annurev.psych.56.091103.07 0225

Rauschecker, J. P., and Scott, S. K. (2009). Maps and streams in the auditory cortex: nonhuman primates illuminate human speech processing. Nat. Neurosci. 12, 718-724. doi: 10.1038/nn.2331

Schulze, K., Gaab, N., and Schlaug, G. (2009). Perceiving pitch absolutely: comparing absolute and relative pitch possessors in a pitch memory task. BMC Neurosci. 10:106. doi: 10.1186/1471-2202-10-106

Schulze, K., and Koelsch, S. (2012). Working memory for speech and music. Ann. N Y Acad. Sci. 1252, 229-236. doi: 10.1111/j.1749-6632.2012. 06447.x

Schulze, K., Mueller, K., and Koelsch, S. (2011a). Neural correlates of strategy use during auditory working memory in musicians and non-musicians. Eur. J. Neurosci. 33, 189-196. doi: 10.1111/j.1460-9568.2010.07470.x

Schulze, K., Zysset, S., Mueller, K., Friederici, A. D., and Koelsch, S. (2011b). Neuroarchitecture of verbal and tonal working memory in nonmusicians and musicians. Hum. Brain Mapp. 32, 771-783. doi: 10.1002/hbm. 21060

Sneve, M. H., Magnussen, S., Alnaes, D., Endestad, T., and D’Esposito, M. (2013). Top-down modulation from inferior frontal junction to FEFs and intraparietal sulcus during short-term memory for visual features. J. Cogn. Neurosci. 25, 1944-1956. doi: 10.1162/jocn_a_00426

Steinke, W. R., Cuddy, L. L., and Jakobson, L. S. (2001). Dissociations among functional subsystems governing melody recognition after righthemisphere damage. Cogn. Neuropsychol. 18, 411-437. doi: 10.1080/026432901 25702

Stewart, L. (2011). Characterizing congenital amusia. Q. J. Exp. Psychol. (Hove) 64, 625-638. doi: 10.1080/17470218.2011.552730

Stewart, L., Overath, T., Warren, J. D., Foxton, J. M., and Griffiths, T. D. (2008). fMRI evidence for a cortical hierarchy of pitch pattern processing. PLoS One 3:e1470. doi: 10.1371/journal.pone.0001470

Stewart, L., von Kriegstein, K., Warren, J. D., and Griffiths, T. D. (2006). Music and the brain: disorders of musical listening. Brain 129, 2533-2553. doi: 10 1093/brain/awl171

Tillmann, B., Albouy, P., and Caclin, A. (2015). "Congenital amusias," in The Auditory System (Handbook of Clinical Neurology), eds G. G. Celesia and G. S. Hickok (Oxford: Elsevier).

Tillmann, B., Janata, P., and Bharucha, J. J. (2003). Activation of the inferior frontal cortex in musical priming. Brain Res. Cogn. Brain. Res. 16, 145-161. doi: 10. 1016/s0926-6410(02)00245-8

Tillmann, B., Koelsch, S., Escoffier, N., Bigand, E., Lalitte, P., Friederici, A. D., et al. (2006). Cognitive priming in sung and instrumental music: activation of inferior frontal cortex. Neuroimage 31, 1771-1782. doi: 10.1016/j.neuroimage.2006. 02.028

Tillmann, B., Schulze, K., and Foxton, J. M. (2009). Congenital amusia: a shortterm memory deficit for non-verbal, but not verbal sounds. Brain Cogn. 71, 259264. doi: 10.1016/j.bandc.2009.08.003

Varela, F., Lachaux, J. P., Rodriguez, E., and Martinerie, J. (2001). The brainweb: phase synchronization and large-scale integration. Nat. Rev. Neurosci. 2, 229239. doi: $10.1038 / 35067550$

Williamson, V. J., McDonald, C., Deutsch, D., Griffiths, T. D., and Stewart, L. (2010). Faster decline of pitch memory over time in congenital amusia. $A d v$. Cogn. Psychol. 6, 15-22. doi: 10.2478/v10053-008-0073-5

Williamson, V. J., and Stewart, L. (2010). Memory for pitch in congenital amusia: beyond a fine-grained pitch discrimination problem. Memory 18, 657-669. doi: 10.1080/09658211.2010.501339 
Williamson, V. J., and Stewart, L. (2013). "Congenital amusia," in Pediatric Neurology, Part I, eds O. Dulac, M. Lassonde and H. B. Sarnat (Newnes: Elsevier), 237-239.

Wolf, R. C., Sambataro, F., Lohr, C., Steinbrink, C., Martin, C., and Vasic, N. (2010). Functional brain network abnormalities during verbal working memory performance in adolescents and young adults with dyslexia. Neuropsychologia 48, 309-318. doi: 10.1016/j.neuropsychologia.2009.09.020

Zatorre, R. J., Belin, P., and Penhune, V. B. (2002). Structure and function of auditory cortex: music and speech. Trends Cogn. Sci. 6, 37-46. doi: 10 . 1016/s1364-6613(00)01816-7

Zatorre, R. J., Evans, A. C., and Meyer, E. (1994). Neural mechanisms underlying melodic perception and memory for pitch. J. Neurosci. 14, 1908-1919.

Zatorre, R. J., and Samson, S. (1991). Role of the right temporal neocortex in retention of pitch in auditory short-term memory. Brain 114(Pt. 6), 2403-2417. doi: 10.1093/brain/114.6.2403
Conflict of Interest Statement: The authors declare that the research was conducted in the absence of any commercial or financial relationships that could be construed as a potential conflict of interest.

Received: 15 October 2014; accepted: 10 January 2015; published online: 04 February 2015.

Citation: Albouy P, Mattout J, Sanchez G, Tillmann B and Caclin A (2015) Altered retrieval of melodic information in congenital amusia: insights from dynamic causal modeling of MEG data. Front. Hum. Neurosci. 9:20. doi: 10.3389/fnhum.2015.00020 This article was submitted to the journal Frontiers in Human Neuroscience.

Copyright (๑) 2015 Albouy, Mattout, Sanchez, Tillmann and Caclin. This is an openaccess article distributed under the terms of the Creative Commons Attribution License (CC BY). The use, distribution and reproduction in other forums is permitted, provided the original author(s) or licensor are credited and that the original publication in this journal is cited, in accordance with accepted academic practice. No use, distribution or reproduction is permitted which does not comply with these terms. 